【研究简报】

\title{
环 $\mathbf{Z} /\left(2^{e}\right)$ 上本原序列最高权 位的 0,1 分布 (II)
}

\author{
戚文峰 周锦君 \\ (郑州信息工程学院应用数学系,郑州 450002)
}

\section{关链词 线性递归序列 本原序列最高权位序列 0,1 分布}

设 $f(x)=x^{n}+c_{n-1} x^{n-1}+\cdots+c_{0}$ 是 $Z /\left(2^{e}\right)$ 上首一多项式, 适合关系式

$$
a_{i+n}=-\left(c_{0} a_{i}+c_{1} a_{i+1}+\cdots+c_{n-1} a_{i+n-1}\right), i=0,1,2, \cdots
$$

的 $Z /\left(2^{e}\right)$ 上序列 $\boldsymbol{a}=\left(a_{0}, a_{1}, \cdots\right)$ 称由 $f(x)$ 生成的线性递归序列, 由 $f(x)$ 生成的 $Z /\left(2^{e}\right)$ 上 的所有序列的集合记为 $G(f(x))_{e}$, 并记 $G^{\prime}(f(x))_{e}=\left\{\boldsymbol{a} \in G(f(x))_{e} \mid \boldsymbol{a} \neq \mathbf{0} \bmod 2\right\}$. 递归式 (1) 等价于关系式 $f(x) \boldsymbol{a}=\mathbf{0}=(0,0, \cdots)$, 其中 $x$ 表示移位算子, 即 $x \boldsymbol{a}=\left(a_{1}, a_{2}, a_{3}, \cdots\right)$. $Z /\left(2^{e}\right)$ 上序列 $\boldsymbol{a}$ 有唯一权位分解 $\boldsymbol{a}=\boldsymbol{a}_{0}+\boldsymbol{a}_{1} 2+\cdots+\boldsymbol{a}_{e-1} 2^{e-1}$, 其中 $\boldsymbol{a}_{i}=\left(a_{i 0}, a_{i 1}, \cdots\right)$ 是 0,1 序列, 并称 $\boldsymbol{a}_{i}$ 是 $\boldsymbol{a}$ 的第 $i$ 权位序列, 称 $\boldsymbol{a}_{e-1}$ 为 $\boldsymbol{a}$ 的最高权位序列.

对 $Z /\left(2^{e}\right)$ 上首一 $n$ 次多项式 $f(x)$, 若 $f(0)$ (即 $c_{0}$ ) 是可逆元, 则由文献 [1], $f(x)$ 的周期 $\operatorname{per}(f(x))_{e} \leqslant 2^{e-1}\left(2^{n}-1\right)$. 当 $\operatorname{per}(f(x))=2^{e-1}\left(2^{n}-1\right)$ 时, 称 $f(x)$ 是 $Z /\left(2^{e}\right)$ 上 $n$ 次本原 多项式, 并称 $G^{\prime}(f(x))$ 中序列为 $f(x)$ 生成的本原序列. 文献 [2]给出了本原多项式的系数 判别条件, 文献 [3] 给出了如下具有重要密码意义的保摘定理: 设 $f(x)$ 是 $Z /\left(2^{e}\right)$ 上 $n$ 次本原 多项式, 对 $\boldsymbol{a}, \boldsymbol{b} \in G(f(x))_{e}$, 则 $\boldsymbol{a}=\boldsymbol{b}$ 当且仅当它们的最高权位序列相等, 即 $\boldsymbol{a}_{e-1}=\boldsymbol{b}_{e-1}$.

设 $f(x)$ 是 $Z /\left(2^{e}\right)$ 上 $n$ 次本原多项式, $\boldsymbol{a} \in G^{\prime}(f(x))_{e}$, 由文献 [4] 可知, $\boldsymbol{a}$ 的第 $k$ 权位序 列 $a_{k}$ 的周期 $\operatorname{per}\left(a_{k}\right)=2^{k} T$, 其中 $T=2^{n}-1$. 由文献 [1]或 [4]知, 对 $1 \leqslant d \leqslant e-1, Z /\left(2^{e}\right)$ 上, $x^{2^{d-1} T}-1=2^{d} h_{d}(x) \bmod f(x)$, 其中 $h_{k}(x)$ 是 $Z /\left(2^{e}\right)$ 上次数小于 $n$ 的多项式且 $h_{k}(x) \neq 0$ $\bmod 2$. 文献 [5]给出如下的结论:

命题 $\mathbf{A}$ 设 $f(x)$ 是 $Z /\left(2^{e}\right)$ 上 $n$ 次本原多项式, $T=2^{n}-1, a \in G^{\prime}(f(x))_{e}, d=[e / 2]$, $\boldsymbol{s}=h(x) \boldsymbol{a} \bmod 2^{d}$, 则 $\boldsymbol{a}_{e-1}$ 中“ “" (或“ 1 ”) 所占的比例 $\lambda$ 为

$$
\frac{1}{2}-\frac{N(s, 0)}{2^{d} T} \leqslant \lambda \leqslant \frac{1}{2}+\frac{N(s, 0)}{2^{d} T},
$$

其中 $N(s, 0)$ 表示 $s$ 在一个周期中“ 0 ”的个数, $h(x)=\left\{\begin{array}{l}h_{d}(x), \text { 若 } e=2 d, \\ h_{d+1}(x), \text { 若 } e=2 d+1 .\end{array}\right.$

利用这一结论, 文献 [5]证明了当 $e$ 足够大时, 绝大部分的 $a_{e-1}$ 的 0,1 分布是很好的. 例 如, 当 $e=32$ 时, 对 $Z /\left(2^{e}\right)$ 上 $n$ 次本原多项式 $f(x), G^{\prime}(f(x))$ e 中满足 $0.498046875 \leqslant \lambda \leqslant$ 0.501953125 的序列 $a$ 至少占 $G^{\prime}(f(x))_{e}$ 中所有序列的 $99.6 \%$. 当 $e$ 大于 32 时结论会更 好.

上面结论中, 对有部分 $a_{e-1}$ 的 0,1 分布没法估计, 这是因为在 $s=h(x) a \bmod 2^{d}$ 的一个 
周期中零的个数很难给出估计, 本文对 $Z /\left(2^{4}\right)$ 上本原序列中零的个数给出一个上界, 从而对 $e \geqslant 8$, 序列 $a_{e-1}$ 在一个周期中“ 0 ”个数所占比例在 $40 \%$ 到 $60 \%$ 之间.

首先把命题 $A$ 推广到如下一般情形:

定理 1 设 $f(x)$ 是 $Z /\left(2^{e}\right)$ 上 $n$ 次本原多项式, $T=2^{n}-1, a \in G(f(x))_{e}$ 且 $a_{0} \neq 0,1 \leqslant$ $d \leqslant e / 2, \boldsymbol{s}=h_{e-d}(x) \boldsymbol{a} \bmod 2^{d}$, 则 $\boldsymbol{a}_{e-1}$ 中“0”(或“1”) 所占的比例 $\lambda$ 为

$$
\frac{1}{2}-\frac{N(s, 0)}{2^{d} T} \leqslant \lambda \leqslant \frac{1}{2}+\frac{N(s, 0)}{2^{d} T},
$$

其中 $N(s, 0)$ 表示 $s$ 在一个周期中“ 0 ”的个数.

引理 1 设 $f(x)$ 是 $F_{2}$ 上 $n$ 次本原多项式, $\boldsymbol{a}=\left(a_{0}, a_{1}, \cdots\right), \boldsymbol{b}=\left(b_{0}, b_{1}, \cdots\right)$ 是由 $f(x)$ 生成的 2 条不同的本原序列 (即是 $m$-序列), 记 $S_{a, b}(u, v)=\left\{i \mid a_{i}=u, b_{i}=v, 0 \leqslant i \leqslant\right.$ $\left.2^{n}-2\right\}, M_{a, b}(u, v)=\left|S_{a, b}(u, v)\right|$, 则 $M_{a, b}(0,0)=2^{n-2}-1, M_{a, b}(0,1)=M_{a, b}(1$, $0)=M_{a, b}(1,1)=2^{n-2}$.

引理 2 设 $f(x)$ 是 $F_{2}$ 上 $n$ 次本原多项式, $n \geqslant 3, \boldsymbol{a}, \boldsymbol{b}, \boldsymbol{c}$ 是由 $f(x)$ 生成的两两不同的 $m$-序列且 $\boldsymbol{a}+\boldsymbol{b}+\boldsymbol{c} \neq \mathbf{0}$, 对 $u, v, w \in F_{2}$, 记 $S_{a, b, c}(u, v, w)=\left\{i \mid a_{i}=u, b_{i}=v, c_{i}=w\right.$, $\left.0 \leqslant i \leqslant 2^{n}-2\right\}$, 令 $M_{a, b, c}(u, v, w)=\left|S_{a, b, c}(u, v, w)\right|$, 则 $M_{a, b, c}(u, v, w)=$ $\begin{cases}2^{n-3}-1, & \text { 若 } u=v=w=0, \\ 2^{n-3}, & \text { 否则. }\end{cases}$

利用引理 1 、引理 2 及权位序列之间的关系得到

引理 3 设 $f(x)$ 是 $Z /\left(2^{4}\right)$ 上 $n$ 次本原多项式, $n \geqslant 3, a$ 是由 $f(x)$ 生成的 $Z /\left(2^{4}\right)$ 上本原 序列, 若 $h_{2}(x) \neq 1 \bmod 2$, 则 $a$ 在一个周期 $\left(=2^{3}\left(2^{n}-1\right)\right)$ 内 “ 0 ”的个数满足

$$
N(a, 0) \leqslant 15 \cdot 2^{n-3}-8 .
$$

由定理 1 和引理 3 得下面的定理 2 .

定理 2 设 $f(x)$ 是 $Z /\left(2^{e}\right)$ 上 $n$ 次本原多项式, $n \geqslant 3, e \geqslant 8, a$ 是由 $f(x)$ 生成的 $Z /\left(2^{e}\right)$ 上本原序列, $h_{2}(x)$ 满足引理 3 的条件, 则 $\boldsymbol{a}_{e-1}$ 中“ “0” (或“1”) 所占的比例满足

$$
\frac{1}{2}-\frac{15 \cdot 2^{n-6}-1}{2\left(2^{n}-1\right)} \leqslant \lambda \leqslant \frac{1}{2}+\frac{15 \cdot 2^{n-6}-1}{2\left(2^{n}-1\right)}
$$

推论 1 条件同定理 2, 则 $a_{e-1}$ 中“0”所占的比例满足 $38.28125 \%<\lambda<61.71875 \%$.

为了更精确估计 $\lambda$, 下面进一步改进引理 3, 为此先给出引理 4 和引理 5 .

引理 4 设 $f(x)$ 是 $F_{2}$ 上 $n$ 次本原多项式, $n \geqslant 4, \boldsymbol{a}, \boldsymbol{b}, \boldsymbol{c}$ 和 $\boldsymbol{d}$ 是由 $f(x)$ 生成的 $m$-序 列, 符号 $M_{\boldsymbol{a}, \boldsymbol{b}, \boldsymbol{c}, \boldsymbol{d}}(u, v, w, x)$ 类似引理 2 中定义, 若 $\boldsymbol{a}, \boldsymbol{b}, \boldsymbol{c}, \boldsymbol{d}$ 两两不同且 $\boldsymbol{a}+\boldsymbol{b}+\boldsymbol{c}, \boldsymbol{a}$ $+b+d, a+c+d, b+c+d$ 和 $a+b+c+d$ 都不为 0 , 则

$$
M_{a, b, c, d}(u, v, w, x)= \begin{cases}2^{n-4}-1, & \text { 若 } u=v=w=0, \\ 2^{n-4}, & \text { 否则. }\end{cases}
$$

引理 $5^{[6]}$ 设 $a$ 是 $Z /\left(2^{e}\right)$ 上 $n$ 次本原序列且 $a \neq 0$, 则 $a$ 在一个周期 $\left(=2\left(2^{n}-1\right)\right)$ 中 0 的个数 $N(a, 0) \leqslant 2^{n}+2^{n / 2}-2$. 
利用引理 4 、引理 5 及权位序列之间的关系得到

引理 6 在引理 3 的条件的基础上, 再假设 $n \geqslant 4, h_{1}(x) h_{2}(x) \neq 1 \bmod (2, f(x)),(1+$ $\left.h_{1}(x)\right) h_{2}(x) \neq 1 \bmod (2, f(x))$, 则 $a$ 在一个周期 $\left(=2^{3}\left(2^{n}-1\right)\right)$ 中 “ 0 ”的个数满足

$$
N(a, 0) \leqslant \min \left\{13 \cdot 2^{n-3}-8,12 \cdot 2^{n-3}+2^{n / 2}-2\right\} .
$$

由定理 1 和引理 6 得下面的定理 3 .

定理 3 设 $f(x)$ 是 $Z /\left(2^{e}\right)$ 上 $n$ 次本原多项式, $n \geqslant 4, e \geqslant 8, a$ 是由 $f(x)$ 生成的 $Z /\left(2^{e}\right)$ 上本原序列且 $a_{0} \neq 0, h_{1}(x)$ 和 $h_{2}(x)$ 满足引理 6 的条件, 则 $\boldsymbol{a}_{e-1}$ 中 “ 0 ” (或 “ 1 ”) 所占的比例满 足

$$
\frac{1}{2}-\frac{6 \cdot 2^{n-4}+2^{\frac{n}{2}-1}-1}{2^{2}\left(2^{n}-1\right)} \leqslant \lambda \leqslant \frac{1}{2}+\frac{6 \cdot 2^{n-4}+2^{\frac{n}{2}-1}-1}{2^{2}\left(2^{n}-1\right)}
$$

且

$$
\frac{1}{2}-\frac{13 \cdot 2^{n-6}-1}{2\left(2^{n}-1\right)} \leqslant \lambda \leqslant \frac{1}{2}+\frac{13 \cdot 2^{n-6}-1}{2\left(2^{n}-1\right)} .
$$

推论 2 条件同定理 3, 则 $a_{e-1}$ 中“0”(或“1”) 所占的比例满足

(i ) 当 $n \leqslant 7$ 时, $40.1579 \%<\lambda<59.8421 \%$;

(ii) 当 $n \geqslant 8$ 时, $40.2505 \%<\lambda<59.7495 \%$;

(iii) 当 $n \geqslant 30$ 时, $40.6249 \%<\lambda<59.3751 \%$.

文献 [5]指出当 $e$ 充分大, 绝大多数的 $a_{e-1}$ 具有好的分布, 推论 2 又保证其他的 $a_{e-1}$ 不会 有太差的分布.

致谢本工作为中国科学院研究生院信息安全国家重点实验室资助项目.

\section{参考文献}

1 Ward M. The arithmetical theory of linear recurring sequences. Trans Amer Math Soc, 1933, 35(6): 600

2 Dai Zongduo, Huang Minqiang. A criterion for primitiveness of polynomials over $Z \bmod 2^{d}$. Chinese Science Bulletin, 1991, 36(11): 892

3 Huang Minqiang, Dai Zongduo. Projective maps of linear recurring sequences with maximal $p$-adic periods. Fibonacci Quart, $1992,30(2): 139$

4 成文峰, 周锦君. 环 $\mathbb{Z} /\left(2^{e}\right)$ 上本原序列最高权位的 0,1 分布. 中国科学, $A$ 辑, 1997, 27(4): 311

5 Dai Zongduo. Binary sequences derived from ML-sequences over rings I: periods and minimal polynomials. Journal of Cryptology, 1990, 5(2): 193

6 Kuzmin A S. The distribution of elements on cycles of linear recurrents over rings of residues. Russian Mathmatical Survey, 1992, 47(6): 219 